\title{
ADMINISTRAÇÃO DE MEDICAMENTOS E A COMUNICAÇÃO DE CONHECIMENTO VIA ONLINE ${ }^{1}$
}

\author{
ON LINE COMMUNICATION OF MEDICINE ADMINISTERING KNOWLEDGE \\ ADMINISTRACIÓN DE MEDICAMENTOS Y LA COMUNICACIÓN DEL \\ CONOCIMIENTO VIA ON LINE
}

\author{
Paulo Celso Prado Telles Filho ${ }^{2}$ \\ Luzia Elaine Galdeano ${ }^{2}$ \\ Adriana Inocenti Miasso ${ }^{2}$ \\ Maria Helena Larcher Calir ${ }^{3}$ \\ Isabel Amélia Costa Mendes ${ }^{4}$
}

\begin{abstract}
RESUMO: O presente estudo tem como objetivo verificar e descrever como tem-se dado a comunicação do conhecimento em enfermagem, via online, acerca da administração de medicamentos. Para o levantamento das páginas eletrônicas utilizou-se do site MetaBusca-Terra (www.terra.com.br). Encontrou-se 166 páginas eletrônicas; no entanto, somente 45 relacionavam-se ao tema, as quais foram analisadas e categorizadas segundo tipos de apresentaçăo, sites, idiomas e endereços eletrônicos. Verificou-se que as mesmas apresentavam caracteristicas em comum, são elas: Artigos ( 18 ), Boletins Informativos ( 8 ) e Propagandas de medicação ( 7 ). Em relaçăo aos sites destacouse o Yahoo, com 34 páginas eletrônicas. $\mathrm{O}$ idioma predominante foi o inglês com 36 páginas. A comunicação do conhecimento via online é fundamental no que tange à administração de medicamentos pois, a partir dela, a difusão do conhecimento dar-se-á de forma mais rápida e produtiva. Indubitavelmente clientes e profissionais receberão incontestáveis benefícios.
\end{abstract}

PALAVRAS-CHAVE: comunicação, conhecimento, enfermagem

\section{INTRODUÇÄO}

O advento da globalização trouxe aos dias atuais um considerável aumento no volume de informações e inovaçōes relativas às diversas áreas do conhecimento, acentuando o papel de destaque da comunicação no processo de pesquisa. Há consenso de que a produção do conhecimento não é suficiente; é uma fase do processo de investigação que deve, necessariamente, ser seguida pela fase de comunicação, sendo esta vista como parte central e prioritária do mesmo (MENDES, 1996).

$\mathrm{O}$ avanço da tecnologia, principalmente no que concerne à tecnologia computacional vem em muito favorecer a comunicação de trabalhos cientificos de todas as áreas do conhecimento, incluindo a enfermagem.

' Trabalho apresentado à disciplina Seminário Avançado de Comunicação do Conhecimento em Enfermagem do Programa de Pós-Graduação em Enfermagem Fundamental da Escola de Enfermagem de Ribeirāo Preto - Universidade de São Paulo (EERP-USP), Centro Colaborador da Organizaçāo Mundial da Saúde para o desenvolvimento da pesquisa em enfermagem. CAPES.

${ }^{2}$ Mestrandos do Programa de Enfermagem Fundamental da EERP - USP. Fonte de auxilio: USP.

${ }^{3}$ Professora Doutora do Departamento de Enfermagem Geral e Especializada da EERP -

- Pesquisador 1 A do CNPq e Professora Titular do Departamento de Enfermagem Geral e Especializada da EERP-USP. 
A revolução digital introduziu facilidades na geração e na disseminação do conhecimento exigindo, para tanto, sistemas de organização, distribuição e seleção(MENDES; TREVISAN; EVORA, 2000). Assim sendo, é importante para esta profissão investir na tecnologia para a divulgação de trabalhos de pesquisa, inserindo-se nas transformações trazidas através da internet e permitindo a ampliação do conhecimento através dos artigos publicados (MONTEIRO, 1999). A Internet vem possibilitar a utilização de um canal permanente de comunicação que não somente permite o intercâmbio de informações, mas também a realização de atividades conjuntas (BLASCO, 1996).

"Percebe-se imediatamente o interesse e o impacto dessa evolução nas aplicações educacionais das tecnologias: de uma prática relativamente solitária, vai-se voltar, graças à conexão, com uma rede poderosa, para um uso mais coletivo e de convivio dessas tecnologias" (LOING, 1998, p. 42).

A difusão das inovações oferece um poderoso paradigma para a contextualização, desenvolvimento e aceitação de projetos de publicações eletrônicas. Vários autores têm afirmado que o desenvolvimento de tais publicações produzirá profundas mudanças na comunicação entre estudiosos (HAHN; $\mathrm{SCHOCH}, 1997$ ).

Em paralelo a esse contexto, a execução da terapêutica medicamentosa, tarefa cotidianamente desenvolvida pela equipe de enfermagem, passa por uma mudança de paradigma; ou seja, não mais basta a realização de tarefas rotineiras, mas sim, necessita-se de conhecimento científico que embase e garanta uma administração de medicamentos segura. Um importante tópico nessa temática, ou seja, erros de medicação, refere-se à ocorrência de erros relacionados ao escasso conhecimento (CARVALHO, 2000).

Portanto, faz-se extremamente interessante a parceria das temáticas de administração de medicamentos e divulgação do conhecimento via online, considerando ser este mecanismo um facilitador do processo de difusão do conhecimento, podendo resultar em maior atualização, competência e eficácia na administração de medicamentos.

\section{OBJETIVO}

O presente estudo tem como objetivo verificar e descrever como tem-se dado a comunicação do conhecimento em enfermagem, via online, acerca da administração de medicamentos.

\section{METODOLOGIA}

Para o levantamento das páginas eletrônicas acessou-se o site MetaBusca-Terra (www.terra.com.br) que oferece, através de seu serviço de busca um elenco acerca dos sites nacionais, americanos, espanhóis, alemães, franceses e italianos.

As palavras-chave foram selecionadas observando-se a adequação à temática proposta e de acordo com a indexação.

Foram realizados 6 cruzamentos utilizando-se das palavras: "administração de medicamentos e enfermagem", "administration medicaments and nursing", "medicamentos e enfermagem", "medicaments and nursing", "drugs and nursing" e "drugs administration and nursing".

Ressalta-se que a palavra "drogas" foi inicialmente utilizada como palavra-chave para busca; no entanto, foram encontradas páginas eletrônicas referentes ao etilismo, tabagismo e drogas ilicitas, desviando-se da temática deste estudo, e sendo portanto descartada dos cruzamentos.

Para todos os cruzamentos estipulou-se como tempo de busca noventa segundos. 
As páginas eletrônicas encontradas foram analisadas e categorizadas segundo tipos de apresentação, sites, idiomas e endereços eletrônicos.

\section{RESULTADOS E DISCUSSÃO}

Inicialmente julgamos de utilidade ao leitor citar algumas definições de terminologias de interesse para este trabalho. São elas: on line - termo utilizado para designar todo tipo de transação entre computadores; homepage - página de entrada de um Web site (o termo pode ser utilizado para indicar a página principal de uma determinada seção); Web site - um servidor da www que contém páginas interligadas, conhecidas como documentos de hipertexto (Documento capaz de incluir em seu conteúdo ligações com outras partes do mesmo documento); WWW ou World Wide Web -área da Internet que contém documentos em formato de hipermidia (uma combinação de hipertexto e multimídia); Site - espécie de "casa" virtual de uma pessoa, empresa ou instituição; um grupo de documentos relacionados e arquivos que residem em um servidor; link - nome dado às imagens ou palavras que dão acesso a outros conteúdos em um documento hipertexto (LUCENA, 1999).

Foram realizados seis cruzamentos devido a dificuldade em encontrar páginas eletrônicas que tivessem relação com a temática abordada. Cumpre esclarecer que a coleta de dados deuse em periodo de 09/09/2000 a 17/09/2000.

Faz-se importante relatar que foram encontradas 166 páginas eletrônicas; no entanto, apenas 45 tinham relação direta com a temática estudada. As demais páginas abordaram temas referentes a administração de serviços de enfermagem, grade curricular de cursos de enfermagem, propagandas farmacêuticas com ênfase em comércio e anestesiologia.

O quadro 1 indica a quantidade de páginas eletrônicas encontradas, relacionadas com a temática, em cada cruzamento.

\section{QUADRO 1 - DISTRIBUIÇÃO DE PÁGINAS ELETRÔNICAS ENCONTRADAS APARTIR DE CRUZAMENTO DE PALAVRAS-CHAVE}

\begin{tabular}{|l|c|}
\hline Cruzamento de palavras-chave & $\begin{array}{c}\text { Quantidade de páginas } \\
\text { eletrônicas }\end{array}$ \\
\hline Administração de medicamento e enfermagem & 01 \\
\hline Administration medicaments and nursing & 09 \\
\hline Medicamentos e enfermagem & 06 \\
\hline Medicaments and Nursing & 04 \\
\hline Drugs and nursing & 16 \\
\hline Drugs administration and nursing & 09 \\
\hline Total & $\mathbf{4 5}$ \\
\hline
\end{tabular}

NOTA: Tabela confeccionada a partir do site Metabusca-Terra

Destaca-se o cruzamento "drugs and nursing" com 16 páginas eletrônicas, seguido pelos cruzamentos "administration medicaments and nursing" e "drugs administration and nursing", contendo nove páginas em cada cruzamento. Os demais cruzamentos mostraram reduzido número de páginas conforme evidenciado pela tabela acima.

É oportuno esclarecer que somente uma página eletrônica repetiu-se, a qual será destacada e comentada no decorrer do estudo.

As páginas encontradas foram distribuidas em quadros para melhor visualização. Destacamos que os quadros respeitam os cruzamentos das palavras.

No cruzamento de palavras "administration medicaments and nursing" encontrou-se as seguintes páginas eletrônicas as quais citam-se os titulos, já traduzidos: Novos estudos sobre 
a incidência e custo da má prática médica (1) ${ }^{5}$; Eficácia da administração de quimioterápicos via oral (2); Caracteristicas de produtos que podem ser administrados através da caneta Britajt (3); Mycophenolate Mofetil (4); Promethazine Hydrochloride (5); Fenobarbital (6); Injeção de Ketorolac Tromethamine (7); Sumário das características dos produtos (8); Um modelo da lei na utilização de narcóticos(9) Enfermagem-assistência à saúde (10).

$\mathrm{O}$ quadro que se segue é referente ao cruzamento "administração de medicamentos e enfermagem" "e "administration medicaments and nursing". É importante ressaltar que o referido quadro envolve dois cruzamentos devido ao fato de no cruzamento das palavras "administração de medicamentos e enfermagem" ter sido encontrada somente uma página eletrônica.

QUADRO 2 - DISTRIBUIÇÃO DE PÁGINAS ELETRÔNICAS ANALISADAS SEGUNDO TIPOS DE APRESENTAÇÃO, SITES, IDIOMAS E ENDEREÇOS ELETRÔNICOS REFERENTES AOS CRUZAMENTOS: “ADMINISTRATION MEDICAMENTS AND NURSING" E "ADMINISTRAÇÃO DE MEDICAMENTOS E ENFERMAGEM"

\begin{tabular}{|c|c|c|c|}
\hline $\begin{array}{c}\text { TIPOS DE } \\
\text { APRESENTAÇÃO }\end{array}$ & SITES & IDIOMAS & ENDEREÇOS ELETRÔNICOS \\
\hline Artigos & $\begin{array}{l}\text { Yahoo } \\
\text { Infoseek }\end{array}$ & $\begin{array}{l}\text { Inglês } \\
\text { Francês }\end{array}$ & $\begin{array}{l}\text { 01- http://www.braytonlow. com } \\
\text { 02- http://www.pslgroup.com }\end{array}$ \\
\hline $\begin{array}{l}\text { Propagandas de } \\
\text { medicamentos }\end{array}$ & Yahoo & Inglês & $\begin{array}{l}\text { 03- http://www.britannia/pharm.co.uk } \\
\text { 04- http://www.btinternet.com } \\
\text { 05- http://www.rxmed.com } \\
\text { 06- http://www.1.mosby.com } \\
\text { 07- http://www.rocheusa.com } \\
\text { 08- http://www.degob.de }\end{array}$ \\
\hline Boletim informativo & Yahoo & Inglês & 09- http://www.undcp.org/hulletin \\
\hline $\begin{array}{l}\text { Propaganda de serviço } \\
\text { de enfermagem }\end{array}$ & Cadê & Português & 10- http://www.rio2001negóci os.com.br \\
\hline
\end{tabular}

NOTA: Os números que antecedem os endereços eletrônicos são correspondentes aos títulos indicados acima.

Observando o quadro 2 verifica-se a existência de dez páginas eletrônicas que em relação ao tipo de apresentação registram seis propagandas de medicação, as quais incluem composição, contra-indicações, precauções, posologia e método de administração; dois artigos incluindo erros de medicação e eficácia da administração de quimioterápicos via oral; um boletim informativo sobre narcóticos e uma propaganda de serviços de enfermagem incluindo administração de medicamentos. Quanto aos sites de busca, há a predominância do Yahoo com oito páginas, seguindo-se os sites Infoseek e Cadê com um registro a cada site. O idioma Inglês destaca-se contando com oito registros e seguindo-se dos idiomas Francês e Português com um cada.

A seguir, apresenta-se a citação dos titulos das páginas eletrônicas encontradas a partir do cruzamento das palavras "medicamentos e enfermagem": Enfermagem assistência à saúde (1); Informação farmacêutica on-line (2) "; Informações médicas em Uberlândia ${ }^{6}$ (3); Informações médicas em Uberlândia e Triângulo Mineiro" (4); Saúde, informações e curiosidades (5) e

\footnotetext{
${ }^{5} \mathrm{~A}$ inclusão desta página justifica-se devido ao fato de embora o titulo referir-se à questões médicas o conteúdo do mesmo reporta-se à temática do presente estudo'.

${ }^{6} \mathrm{~A}$ inclusão destas páginas justifica-se devido ao fato de que, embora os títulos não referiremse à temática em estudo, o conteúdo dos mesmos reporta-se à temática da presente investigação².
} 
TELLES FILHO, Paulo Celso Prado et al.

Novos medicamentos contra HIV (6).

QUADRO 3-DISTRIBUIÇÃO DE PÁGINAS ELETRÔNICAS ANALISADAS SEGUNDO TIPOS DE APRESENTAÇĀO, SITES, IDIOMAS E ENDEREÇOS ELETRÔNICOS REFERENTES AO CRUZAMENTO: "MEDICAMENTOS E ENFERMAGEM"

\begin{tabular}{|c|c|c|c|}
\hline $\begin{array}{c}\text { TIPOS DE } \\
\text { APRESENTAÇÃO }\end{array}$ & SITES & IDIOMAS & ENDEREÇOS ELETRŌNICOS \\
\hline $\begin{array}{l}\text { Propaganda de serviço } \\
\text { de enfermagem }\end{array}$ & Cadê & Português & 1- http://www.rio2001negócios.com.br \\
\hline Boletins informativos & $\begin{array}{l}\text { Infoseek } \\
\text { Zeek e } \\
\text { Aonde } \\
\text { Cadê e } \\
\text { La } \\
\text { Brújula } \\
\text { Infoseek }\end{array}$ & $\begin{array}{l}\text { Português } \\
\text { Português } \\
\text { Português } \\
\text { Espanhol }\end{array}$ & $\begin{array}{l}\text { 2- http://www.netpage.em.com.br } \\
3 \text { e 4- http://medhall.hypermart.net } \\
\text { 5- http://geocities.combusca.brujula.cl } \\
\text { 6-http://www.projinf.org/spanish/ }\end{array}$ \\
\hline
\end{tabular}

NOTA: Os números que antecedem os endereços eletrônicos são correspondentes aos titulos indicados acima.

Neste quadro os tipos de apresentação encontrados são: uma página contendo propaganda de serviço de enfermagem incluindo a administração de medicamentos e cinco boletins informativos contendo administração, cuidados, textos e informaçōes médicas sobre medicamentos. Ressalta-se a existência de duas páginas em um mesmo endereço eletrônico (Informaçōes médicas em Uberlândia e Informações médicas em Uberlândia e Triângulo Mineiro). Nos sites Cadê e Infoseek localizam-se duas páginas em cada, enquanto nos sites Zeek e La Brújula verificou-se a presença de uma página em cada. Destaca-se a existência de páginas que localizam-se em dois sites. Em relação ao idioma, uma página encontra-se em espanhol, sendo as quatro páginas iniciais encontradas em lingua portuguesa.

A seguir, registram-se os titulos das páginas eletrônicas referentes ao cruzamento das palavras: "medicaments and nursing": Novos estudos sobre a incidência e custo da má prática médica (1); Um modelo da lei na utilização de narcóticos (2); Composição e substâncias de um medicamento (3) e Novos livros e vídeos (4).

QUADRO 4 - DISTRIBUIÇÃO DE PÁGINAS ELETRÔNICAS ANALISADAS SEGUNDO TIPOS DE APRESENTAÇÃO, SITES, IDIOMAS E ENDEREÇOS ELETROONICOS REFERENTES AO CRUZAMENTO DAS PALAVRAS: "MEDICAMENTS AND NURSING"

\begin{tabular}{|l|l|l|l|}
\hline \multicolumn{1}{c|}{$\begin{array}{c}\text { TIPOS DE } \\
\text { APRESENTAÇÃO }\end{array}$} & \multicolumn{1}{|c|}{ SITES } & \multicolumn{1}{c|}{ IDIOMAS } & \multicolumn{1}{c|}{ ENDEREÇOS ELETRŌNICOS } \\
\hline Artigo & Yahoo & Inglês & 1- http://www.braytonlow.com \\
\hline Boletins Informativos & Yahoo & Inglês & 2- http://www.undcp.org/hulletin \\
& Yahoo & Francês & 3- http://www.med.univ-rennes1fr \\
\hline Propaganda de livros & Yahoo & Inglês & 4- http://www.ass.edu/depts/libray \\
\hline
\end{tabular}

NOTA: Os números que antecedem os endereços eletrônicos são correspondentes aos titulos indicados acima. 
Identificam-se quatro páginas eletrônicas, todas localizadas no site Yahoo e assim distribuídas segundo idioma: três em Inglês e uma em Francês.

Quanto ao conteúdo, verificou-se uma página composta por um artigo acerca da má prática profissional incluindo erros de medicação (o qual repete-se no primeiro quadro), dois boletins informativos (o primeiro sobre narcóticos e o segundo relacionado à princípios ativos dos medicamentos) e um site de propaganda de livros e vídeos relacionados à temática.

Em seqüência seguem-se os títulos das páginas encontradas com o cruzamento das palavras "drugs and nursing": Links de medicamentos comumente usados (1); Drogas antiepiléticas e enfermagem (2); Uso apropriado de drogas psicotrópicas em enfermagem domiciliar(3); Medicamentos e prática de enfermagem: um guia de A-Z (4); Enfermagem virtual: drogas e medicamentos (5); Farmacologia em enfermagem (6); Farmácia, farmacêuticos e prescrição de medicamentos(7); Saúde atual, raça e idade, interferindo na prescrição de medicamentos (8); Psiquiatria, arquivos de enfermagem: drogas ilicitas (9); Pesquisas e artigos, segurança com medicamentos comumente utilizados em puérperas (10); Medicamentos e implicações de enfermagem (11), Medicamentos pediátricos e implicações de enfermagem: guia rápido de administração de medicamentos pediátricos (12); Lista de medicamentos recentemente aprovados (13); Possiveis efeitos de alguns medicamentos no puerpério (14); Midazolam (15) Medicamentos mais seguros utilizados em uma maternidade (16).

QUADRO 5 - DISTRIBUIÇÃO DE PÁGINAS ELETRÔNICAS ANALISADAS SEGUNDO TIPOS DE APRESENTAÇÃO, SITES, IDIOMAS E ENDEREÇOS ELETRÔNICOS REFERENTES AO CRUZAMENTO DAS PALAVRAS: "DRUGS AND NURSING"

\begin{tabular}{|c|c|c|c|}
\hline $\begin{array}{c}\text { TIPOS DE } \\
\text { APRESENTAÇÃO }\end{array}$ & SITES & IDIOMAS & ENDEREÇOS ELETRŌNICOS \\
\hline $\begin{array}{l}\text { Lista de Links sobre } \\
\text { medicamentos }\end{array}$ & Yahoo & Inglês & 01- http://www.nursefriendly.com/nursing/drugs \\
\hline Artigos & Yahoo & & $\begin{array}{l}\text { 02- http://neuro-www.mgh.harvard.Edu } \\
\text { 03- http://www.aafp.org/afp/20000301/1437.html } \\
\text { 04- http://www.donfer.co.uk } \\
\text { 05- http://www.virtualnurse.com/nursing/drugs. } \\
\text { 06- http://www.merritt.edu/adn/pharm.html } \\
\text { 07- http://www.aslme.org/publications/indexes } \\
\text { 08- http://www.ahcpr.gov/research } \\
\text { 09- http://www.mailbase.ac.uk } \\
\text { 10- http://webmd.lycos.com/content/article }\end{array}$ \\
\hline $\begin{array}{l}\text { Propaganda } \\
\text { (serviços livros, } \\
\text { softwares) }\end{array}$ & Yahoo & Inglês & $\begin{array}{l}\text { 11- http://store.yahoo.com/medtech } \\
\text { 12- http://www.arkanar.minsk.by/ }\end{array}$ \\
\hline $\begin{array}{l}\text { Páginas eletrônicas } \\
\text { de Universidades e } \\
\text { Hospitais }\end{array}$ & Yahoo & Inglês & $\begin{array}{l}\text { 13- http://www1.mosby.com/MERLIN/ } \\
\text { 14- http://www.health.ucsd.edu/pharmacy } \\
\text { 15- http://www.aap.org }\end{array}$ \\
\hline $\begin{array}{l}\text { Propaganda de } \\
\text { medicamento }\end{array}$ & Yahoo & Inglês & 16-http://www.nursing.uiowa.du/sites/pedspain \\
\hline
\end{tabular}

NOTA: Os números que antecedem os endereços eletrônicos são correspondentes aos titulos indicados acima.

O quadro 5 mostra 16 páginas eletrônicas, sendo uma referente a Links sobre medicamentos, nove artigos referentes a métodos de administração, farmacologia, psiquiatria, pediatria e pesquisa em enfermagem. Foram encontradas também duas páginas que contém propagandas de livros referentes à temática, três páginas de Universidades e Hospitais e uma 
propaganda de medicamentos. A totalidade das páginas localiza-se nos sites Yahoo em idioma Inglês.

Finalizando, realizou-se outro cruzamento, com as palavras "drugs administration and nursing" em que os titulos assim descrevem-se: Orientação de enfermagem: administração de medicamentos (1); Caminhos alternativos na administração de medicamentos: vantagens e desvantagens (2); Manejo da dor no pós-operátório: uso do Tramadol (3); Conduzindo a terapia medicamentosa de pacientes que estão recebendo nutrição enteral ou parenteral (TPN) (4); Controle ocupacional à exposição de drogas de risco(5); Segurança nos sistemas de administração de medicamentos intravenosos (6); Pílulas e cápsulas mastigáveis (7); Centro de avaliação e pesquisa de medicamentos (8) e Cursos atuais dentro da gradıação: farmacologia em enfermagem (9).

A seguir apresentam-se as páginas segundo o tipos de apresentação, sites, idiomas em que se encontram e endereços eletrônicos.

QUADRO 6: DISTRIBUIÇÃO DE PÁGINAS ELETRÔNICAS ANALISADAS SEGUNDO TIPOS DE APRESENTAÇÃO, SITES, IDIOMAS E ENDEREÇOS ELETRÔNICOS REFERENTES AO CRUZAMENTO DAS PALAVRAS: "DRUGS ADMINISTRATION AND NURSING"

\begin{tabular}{|c|c|c|c|}
\hline $\begin{array}{c}\text { TIPOS DE } \\
\text { APRESENTAÇÃO }\end{array}$ & SITES & IDIOMAS & ENDEREÇOS ELETRÕNICOS \\
\hline Artigos & $\begin{array}{l}\text { Yahoo } \\
\text { Infoseek } \\
\text { IITrovatore } \\
\text { Yahoo } \\
\text { Yahoo } \\
\text { Infoseek }\end{array}$ & $\begin{array}{l}\text { Inglês } \\
\text { Inglês } \\
\text { Inglês } \\
\text { Inglês } \\
\text { Inglês } \\
\text { Inglês }\end{array}$ & $\begin{array}{l}\text { 1- http://www.med.jhu.Edu/nursing } \\
\text { 2- http://www.aap.org/policy } \\
\text { 3- http://www.salus.it/dol/postop } \\
\text { 4-http://www.pharmj.com//Hospital } \\
\text { 5-http://www.osha-slc.gov/dts/osta/otm } \\
\text { 6-http://www.pharmacy.ohio -state.edu/ }\end{array}$ \\
\hline $\begin{array}{l}\text { Lista de Links sobre } \\
\text { medicamentos }\end{array}$ & $\begin{array}{l}\text { Yahoo } \\
\text { Yahoo }\end{array}$ & $\begin{array}{l}\text { Inglês } \\
\text { Inglês }\end{array}$ & $\begin{array}{l}\text { 7- http://www.nursefriendly.com.nursing/drugs } \\
\text { 8- http://www.healthfinder.gov/text/orgs }\end{array}$ \\
\hline $\begin{array}{l}\text { Propaganda de } \\
\text { curso de } \\
\text { enfermagem }\end{array}$ & Yahoo & Inglês & 9-http://www.coninfo.nursing.uiowa.edu/ \\
\hline
\end{tabular}

NOTA: Os números que antecedem os endereços eletrônicos são correspondentes aos titulos indicados acima.

Neste quadro os tipos de apresentação são: seis páginas contendo artigos relacionados à orientação de enfermagem, caminhos alternativos, manejo da dor, drogas de risco, segurança em sistemas intravenosos de administração de medicamentos bem como nutrição enteral e parenteral, duas listas de Links sobre medicamentos e uma propaganda de curso de enfermagem. O site Yahoo é o que predomina com seis páginas, o site Infoseek segue em segundo plano com duas páginas e II trovatore com uma página eletrônica. Quanto ao idioma, a totalidade das páginas encontra-se em inglês.

\section{CONSIDERAÇÕES FINAIS}

Faz-se importante ressaltar as dificuldades no decorrer da presente pesquisa. São elas: pouca familiaridade com o método de busca utilizado, restrições decorrentes do idioma predominante nos achados e principalmente pelo fato de as páginas encontradas apresentarem pouca relação com a temática estudada nos vários cruzamentos. 
Entretanto, os beneficios de sua realização suplantam tais dificuldades. Através da verificação e descrição das páginas eletrônicas referentes aos diversos cruzamentos realizados nesta pesquisa pôde-se detectar como tem se dado a comunicação do conheciimento, via online, acerca da administração de medicamentos em enfermagem. A reflexão de que, na atualidade, é primordial a habilidade no manuseio do método utilizado para a coleta de dados da presente pesquisa foi um aprendizado verdadeiramente útil, pois a projeção futura é de aumento deste tipo de método de comunicação do conhecimento.

Importa relatar que encontrou-se 166 páginas eletrônicas; no entanto, somente 45 relacionaram-se ao tema. Tais páginas, analisadas sob a forma de quadros, apresentaram características em comum. São elas: Artigos ( 18 páginas ), Boletins Informativos ( 8 ) e Propagandas de medicação ( 7 ). Em relação aos sites destaca-se o Yahoo, com 34 páginas eletrônicas. O idioma predominante é o inglês com 36 páginas.

Os serviços de saúde, atualmente, exigem um processo de administração de medicamentos com nivel de excelência. A observação da prática hospitalar, bem como da literatura, tem apontado direta relação entre conhecimento e qualidade da prestação de serviços.

Nesse sentido, a comunicação do conhecimento via online é fundamental pois, a partir dela, a difusão do conhecimento dar-se-á de forma mais rápida e produtiva. Indubitavelmente clientes e profissionais receberão incontestáveis beneficios.

O desenvolvimento e os resultados do presente estudo nos induzem a recomendar o aprendizado dessa atual tecnologia, assim como que se promovam ações facilitadoras para o acesso ao conteúdo referente ao processo da administração de medicameritos via online.

ABSTRACT: The present study aims at verifying and describing how on-line communication of nursing knowledge concerning administration of medication is taking place. In order to assess the websites, the search tool Meta Busca-Terra (www.terra.com.br) was used. One hundred and sixty-six websites were found; however, only 45 were related to the topic. These were analyzed and categorized according to types of presentation, websites, languages, and homepages. It was verified that they presented common characteristics, such as: Articles (18), Informative Bulletins (8) and Medication Advertisements (7). Regarding the websites, Yahoo distinguished from the others, since it featured 34 websites. The predominant language was English, which was found in 36 websites. The on-line communication of knowledge is fundamental in regard to administration of medication for it allows the dissemination of knowledge at a faster and more productive pace. Undoubtedly, clients and professionals will be highly benefited.

KEYWORDS: communication, knowledge, nursing

RESUMEN: El presente estudio tiene como objetivo verificar y describir como se da la comunicación en enfermería de las informaciones más recientes sobre la administración dí medicamentos a través del sistema de internet. Para el levantamiento de las páginas electrónicas se utilizó el sitio MetaBusca-Terra (www.terra.com.br).Se encontraron 166 páginas, pero sólo 45 correspondian al tema. Después de analizadas y categorizadas se identificaron 18 Articulos, 8 Boletines Informativos y 7 Propagandas de medicación. Respecto a los sitios sobresale el Yahoo, con 34 páginas. El idioma predominante es el inglés, con un total de 36 páginas. La comunicación del conocimiento via on-line es fundamental en lo que se refiere a la administración de medicamentos, pues permite la difusión de la información de modo más rápido y eficaz. No cabe duda de que tanto los clientes como los profesionales recibirán incontestables beneficios.

PALABRAS CLAVE: comunicación, conocimiento-información, enfermeria 


\section{REFERÊNCIAS BIBLIOGRÁFICAS}

BLASCO, S. P. Instituto Cohen para el uso correcto de los medicamentos y productos sanitarios. Errores de medicación. II curso sobre administración de medicamentos. Alicante-Espanha, p. 2541, maio, 1996.

CARVALHO, V. T. Erros na administração de medicamentos: análise de relatos dos profissionais de enfermagem. Ribeirão Preto. 2000. 131p. Dissertação (Mestrado) - Escola de Enfermagem de Ribeirão Preto, Universidade de São Paulo.

HAHN, K. L.; SCHOCH, N. A. Applying diffusion theory to electronic publishing: A conceptual framework for examining issues and outcomes. (On line). Available: http://www.asis.org/annual-97/hahnk.h

LOING, B. Escola e tecnologias: reflexão para uma abordagem racionalizada. Rev. Tecnologia Educacional, v.26, n.142, p. 40 - 43, jul./set. 1998.

LUCENA, R. Guia da Internet. Folha de São Paulo, 1999.

MENDES, I. A. C. Enfermagem, conhecimento e comunicação. Rev. Lat-Americana de Enfermagem, Ribeirão Preto, v.4, p. 1-2, abr. 1996. Especial.

MENDES, I. A. C.; TREVIZAN, M. A. ; EVORA, Y. D. M. Comunicação e enfermagem: tendências e desafios para o próximo milênio. Esc. Anna Nery - R. de Enferm, Rio de Janeiro, v.4, n.2, p.217-224, ago. 2000 .

MONTEIRO, A. B. C. et al. Revistas eletrônicas disponiveis que podem contribuir para a divulgação do conhecimento em saúde. Rev. Lat-Americana de Enfermagem, Ribeirão Preto, v.7, n.2. p.87-88, abr. 1999.

Recebido em maio de 2001

Aprovado em setembro de 2001 